\title{
THE APPLICATION OF LATTICE THEORY TO INTEGRATION
}

\section{H. M. MACNEILLE}

As Professor Menger has already observed, one of the purposes of abstraction is to unite apparently diverse bodies of knowledge under a single theory. I shall illustrate this point by showing that the construction of the number system and the theories of measure and integration can be subsumed under a single abstract development in which partially ordered sets and Boolean rings play important roles.

The number system is usually constructed from the positive integers in four steps which yield successively the positive rationals, positive reals, all real numbers, and complex numbers. With the exception of the second step, these extensions are obtained by defining elements of a particular set as ordered pairs of elements of the preceding set. Though differing in details, these constructions are much alike. The construction of the positive reals from the positive rationals, however, is quite different. Of the four common methods of making this extension we shall be concerned with the methods of fundamental sequences and sequences of nested intervals. The methods of monotone sequences and Dedekind cuts will not be discussed.

The general procedure in developing an abstract theory from a particular example is to observe just which properties of the example are needed to prove the desired theorems. Then an abstract set with these properties is postulated, and the proofs go through as in the given example. This is not always as easy to do as it sounds, for some properties may be most useful but not necessary in proving theorems. It is just these properties which must be eliminated if the abstraction is to be significant. In general, the effectiveness of an abstraction can be judged by the multiplicity and diversity of the theories subsumed under it.

We now apply this procedure to the construction of the number system. Roughly speaking, the properties of numbers needed for these constructions are the elementary laws relating to sums, products, absolute values, and order. We observe that, although the ordering relation for real numbers is linear (that is, if $a$ and $b$ are real numbers, either $a \leqq b$ or $b \leqq a$ ), only a partial order is defined for complex numbers. This suggests, as turns out to be the case, that the linearity of the order is one of the properties which are useful, but not necessary, 
for the constructions under consideration. Consequently, only partial, not linear, order is postulated for the abstract constructions.

As a matter of fact, the restriction to linear types which characterizes practically all except recent work on order is unnecessary for a great majority of the theorems. In particular, the definitions of least upper and greatest lower bounds do not require that the order be linear. Moreover, in partially ordered sets, these concepts become much more significant, and their study, as Mr. Birkhoff has pointed out, is the theory of lattices. Concepts defined in terms of least upper and greatest lower bounds such as limit superior, limit inferior, and limit apply as well to partial as to linear order. Professor Veblen* has shown that the linear continuum can be defined in terms of order alone. However, the continuity of an ordered set does not require linearity; so, for instance, the theory of Dedekind cuts applies as well to partial as to linear order. Furthermore, the definitions of immediate successors and predecessors and of first and last elements do not depend on the linearity of the order. Professor Ore $\nmid$ has investigated well-ordering in partially ordered sets.

In the euclidean plane, any set with finite Lebesgue measure can be approximated arbitrarily closely by a finite set of rectangles with rational corners. That is, among sets of finite measure the sets formed by a finite number of rational rectangles are dense and denumerable and so may reasonably be expected to be analogous in the theory of measure to the rational numbers. However, if the construction of measurable sets from rational rectangles is to be subsumed under our abstraction from the number system, analogs must be found for addition, multiplication, absolute value, and order. This is done as follows: the sum of two elements is the point set symmetric difference, that is, the points in either but not both of the summands; multiplication is point set intersection; the ordering relation, point set inclusion; and the absolute value, area. If the rectangles are taken half open and the void set is admitted, this addition and multiplication can always be performed within the system. In fact, the set of rational rectangular figures is a Boolean ring of point sets such as Professor Stone has discussed. This system has all the properties of the number system essential to the construction of the positive real numbers from the positive rationals, and so is a realization of our abstract construction.

* $\mathrm{O}$. Veblen, Definitions in terms of order alone in the linear continuum and in wellordered sets, Transactions of this Society, vol. 6 (1905), pp. 165-171.

$\dagger$ O. Ore, On the foundations of abstract algebra. I, Annals of Mathematics, (2), vol. 36 (1935), pp. 406-437. 
The abstract construction derived from the method of fundamental sequences is not equivalent to that obtained by the method of sequences of nested intervals. The method of fundamental sequences extends the Boolean ring of rectangular figures to the Boolean ring of sets with finite Lebesgue measure reduced by the ideal of sets of measure zero. The method of sequences of nested intervals extends the Boolean ring of rectangular figures to the ring of sets with finite content reduced by the ideal of sets of content zero. These arguments are independent of the dimensionality of the space. Integration of positive functions may be introduced by considering ordinate sets. In the first case, the integration is that of Lebesgue, in the second case, that of Riemann.* Furthermore, the constructions by which integration is extended to functions with both positive and negative values and to complex-valued functions, and the corresponding constructions for the number system, can be subsumed under a common abstract theory.

\section{HARVARD UNIVERSITY}

* H. M. MacNeille, Extensions of measure, Proceedings of the National Academy of Sciences, vol. 24 (1938), pp. 188-193. 